\title{
Upregulated LMO1 in prostate cancer acts as a novel coactivator of the androgen receptor
}

\author{
HUI GU, TONG LIU, XINZE CAI, YUXIN TONG, YAN LI, CHUNYU WANG and FENG LI \\ Department of Cell Biology, Key Laboratory of Cell Biology, Ministry of Public Health, and Key Laboratory of \\ Medical Cell Biology, Ministry of Education, China Medical University, Shenyang, Liaoning, P.R. China
}

Received July 13, 2015; Accepted August 21, 2015

DOI: $10.3892 /$ ijo.2015.3195

\begin{abstract}
LMO1, a nuclear transcription coregulator, is implicated in the pathogenesis of T-cell acute lymphoblastic leukemia and neuroblastoma. However, the role of LMO1 in human prostate cancer $(\mathrm{PCa})$ is still unknown. Androgen receptor (AR) plays a critical role in the progression of prostate cancer. The activation of AR signaling pathway could be modulated by AR cofactors. In the present study, we discovered that LMO1 could bind to AR and co-localize with AR in the nucleus. In addition, the expression of LMO1 in human PCa tissues was significantly higher than that in benign prostate hyperplasia (BPH) tissues. Moreover, LMO1 appeared to be a novel coactivator to enhance AR transcriptional activities, followed by the elevation of expression of P21 and PSA, downstream targets of AR. Taken together, LMO1 appears to be a coactivator of AR involved in the progression of prostate cancer, and could be a promising molecular target for treating prostate cancer.
\end{abstract}

\section{Introduction}

PCa is the most commonly diagnosed invasive cancer and second only to lung cancer as a cause of cancer death in males in the United States and other Western countries (1). The growth of PCa cells depends on the presence of androgens, a group of steroid hormones. Most prostate cancers are androgen-dependent and respond to the anti-androgens or androgen-deprivation therapy. Unfortunately, patients who have undergone androgen ablation therapy frequently develop a hormone-refractory state, for which there is no curative therapy (2).

AR signaling plays a crucial role in the development of androgen-independent prostate cancer. AR, a member of

Correspondence to: Dr Feng Li, Department of Cell Biology, Key Laboratory of Cell Biology, Ministry of Public Health, and Key Laboratory of Medical Cell Biology, Ministry of Education, China Medical University, Shenyang, Liaoning, P.R. China

E-mail: fli@mail.cmu.edu.cn

Key words: androgen receptor, LMO1, prostate cancer, coactivator the steroid receptor subfamily of nuclear receptors, is a ligand-activated transcription factor that regulates the expression of target genes to mediate the biological function of androgens (3). AR is composed of three major domains: an NH2-terminal transcriptional activation domain (NTD), a central DNA-binding domain (DBD) and a $\mathrm{COOH}$-terminal ligand-binding domain (LBD) (4). After binding to androgen, AR formats the receptor homodimerization and translocates to the nucleus. By recruiting to androgen response elements located within AR-target genes, it can regulate the expression of AR-target genes.

Once activated by a ligand, AR interacts with various cofactors that facilitate transcription according to the general transcriptional machinery (5). Deregulation of AR activity by positive and negative cofactors is likely to play key a key role in prostate cancer progression (6). It has been reported that aberrant regulation of coregulator expression or activity allows weak androgens to activate AR target genes responsible for cell growth, eventually leading to the development of hormone-refractory prostate cancers (7). An increasing number of AR-binding proteins have been recognized to date, however, details remain largely elusive. Therefore, finding novel and more effective cofactors of AR signaling is of great interest in the present research.

The LIM-only (LMO) proteins are a family of nuclear transcription coregulators, which are characterized by the exclusive presence of two tandem LIM domains and no other functional domains (8). The LIM domain is an $~ 55$-residue, highly conserved cysteine-rich zinc-binding motif $(9,10)$. To date, four LMO proteins (LMO1-LMO4) have been identified. These proteins are required for many developmental processes, and are implicated in the initiation and progression of several cancers, including $\mathrm{T}$ cell leukemia, breast cancer and neuroblastoma (11). LMO1 was initially found as T cell oncogene through its association with chromosomal translocations occurring in T-cell acute lymphoblastic leukemia (12). Although LMO1 has been implicated in cancer, most previous studies have only focused on defining its role in T-cell acute lymphoblastic leukemia and neuroblastoma pathogenesis.

This is the first report on the investigation into the role of LMO1 as a potential AR-interacting protein and its function in the regulation of AR transcriptional activity in PCa cells. It has been found that LMO1 expression is significantly increased with a higher level of aggressiveness in human PCa. Together, 
Table I. The sequences of primers used in generating various constructs.

\begin{tabular}{lll}
\hline Primers name & & \multicolumn{2}{c}{ Primer sequences } \\
\hline LMO1 & F: GACACGGAGGGATCCAGATGATGGTGCTGGAC & R: CCAGGCGCGAATTCTTACTGAACTTGGGATTC \\
LMO1 (1-83) & F: GACACGGAGGGATCCAGATGATGGTGCTGGAC & R: GTTCCCTGGAATTCCAAAGAGCCTC \\
LMO1 (24-83) & F: GAAGCGGATCCCCTGTGCGGGCTGTAAC & R: GTTCCCTGGAATTCCAAAGAGCCTC \\
LMO1 (83-156) & F: CTACCGGATCCTCTTTGGCACCACAG & R: CCAGGCGCGAATTCTTACTGAACTTGGGATTC \\
LMO1 (88-147) & F: GCACCAGGATCCACTGTGCTGCTTGC & R: AGGTGCGAATTCCCTGCCCTTCCTC \\
LMO1 (24-147) & F: GAAGCGGATCCCCTGTGCGGGCTGTAAC & R: AGGTGCGAATTCCCTGCCCTTCCTC \\
FLAG- LMO1 & F: CGGAGGGAATTCGATGATGGTGCTGGAC & R: GGCAGGGGATCCTTACTGAACTTGGGATTC \\
AR & F: CCAAGGATCCGGATGGAAGTGCAGTTAGGGC & R: TCCAATCTCGAGCTGGGTGTGGAAATAGATG \\
AR (1-559) & F: CCAAGGATCCGGATGGAAGTGCAGTTAGGGC & R: CTCCACAGAATTCGCAGGTCTTCTGGGG \\
AR (505-676) & F: ATGTGTGGATCCCTGGCGGCATGGTG & R: CAATGGCGAATTCGACATTCAGAAAGATG \\
AR (633-919) & F: CCGGAGGATCCAGAAACTTGGTAATC & R: CTCCACAGAATTCGCAGGTCTTCTGGGG
\end{tabular}

F, forward; R, reverse.

our data suggest that LMO1, as a novel coactivator of AR, can form a complex with $\mathrm{AR}$ and activate AR transcriptional activity in the progression of human prostate cancer.

\section{Materials and methods}

Plasmid construction. AR, ARE-luc and PSA-luc were presented by Dr Yue Zhao (China Medical University). GST-tagged, GFP-tagged, Myc/His-tagged and Flag-tagged LMO1 (full-length and deletions) were constructed by PCR amplification and subcloned into pGEX-5X-1/2 (GE Healthcare, Piscataway, NJ, USA), pEGFP-C1 (Clontech Laboratories, Mountain View, CA, USA), pcDNA3.1Myc/HisA (Invitrogen, San Diego, CA, USA) and p3xFlag CMV (SigmaAldrich, St. Louis, MO, USA) vectors, respectively. All primer sequences for generating these constructs are provided in Table I.

Cell culture and transfections. HEK-293, CV1 and PC-3 cell lines were cultured in Dulbecco's modified Eagle's medium (DMEM) containing 10\% fetal bovine serum (FBS). The prostate CWR22RV1 cells, were cultured in RPMI-1640 medium containing $10 \%$ FBS. Charcoal-stripped serum (CSS) was purchased from HyClone (Thermo Fisher Scientific, Waltham, MA, USA). Lipofectamine 2000 (Invitrogen) was used for transfection. Cells at $\sim 60 \%$ confluence were transfected for $6 \mathrm{~h}$ and incubated with phenol red-free medium containing $10 \% \mathrm{CSS}$ for $16 \mathrm{~h}$. Cell extracts were prepared following another 16-h treatment with $10 \mathrm{nM}$ dihydrotestosterone (DHT) (Sigma-Aldrich). LMO1-siRNA and control siRNA were purchased from Shanghai GeneChem Co. (Shanghai, China).

GST pull-down assay. In vitro GST pull-down has been previously described in detail (13). In vitro transcription and translation of LMO1 proteins were performed by using the TNT-coupled transcription and translation system (Promega Biotech Co., Madison, WI, USA). Equal amounts of GST, GST-fusion proteins immobilized by GST Sepharose Beads
(Amersham Biosciences) were incubated with in vitro translated protein at $4^{\circ} \mathrm{C}$ for $2 \mathrm{~h}$. Protein bound to beads was eluted by $2 \mathrm{X}$ SDS-loading buffers and analyzed using western blot analyses.

Immunoprecipitation and western blot analysis. The cells were washed with cold PBS twice before being lysed in IP lysis buffer supplemented with PMSF and protease inhibitor cocktail (Roche, Basel, Switzerland). Cell lysates were collected, washed and incubated at $4^{\circ} \mathrm{C}$. Then, protein A agarose slurry (GE Healthcare) preloaded with antibodies or normal IgG was added to an equal amount of cell extracts and incubated overnight at $4^{\circ} \mathrm{C}$. Washed precipitated proteins were analyzed by western blot analysis.

Denatured protein were analyzed by SDS-PAGE and transferred to a PVDF membrane (Millipore, Billerica, MA, USA). Samples were incubated and detected with indicated antibodies. Anti-AR, anti-PSA and anti-P21 antibodies were from Thermo Fisher Scientific and anti-LMO1 antibody was from Abcam (Cambridge, MA, USA). Anti-myc antibody was purchased from Santa Cruz Biotechnology (Santa Cruz, CA, USA), anti-Flag antibody was from Shanghai Genomics, Inc., (Shanghai, China) and anti-GAPDH antibody was from Shanghai KangChen (Shanghai, China).

Immunofluorescence staining. Cells were fixed in 4\% paraformaldehyde and permeabilized with $0.2 \%$ Triton $X$. Then they were blocked with normal goat serum, after which they were incubated with the primary antibody, followed by incubation with Alexa Flour 546 secondary antibody (Molecular Probes, Eugene, OR, USA). Nuclei were visualized by TO-PRO-3 (Molecular Probes) and the stained cells were observed using a Leica/Olympus laser confocal scanning microscope.

Tissue samples and immunohistochemistry. Tissue specimens were collected from 97 patients with $\mathrm{PCa}$ who underwent surgery in Shengjing Hospital and First Hospital of China Medical University. All human tissues were collected and used according to protocols approved by the Ethics Committee of 

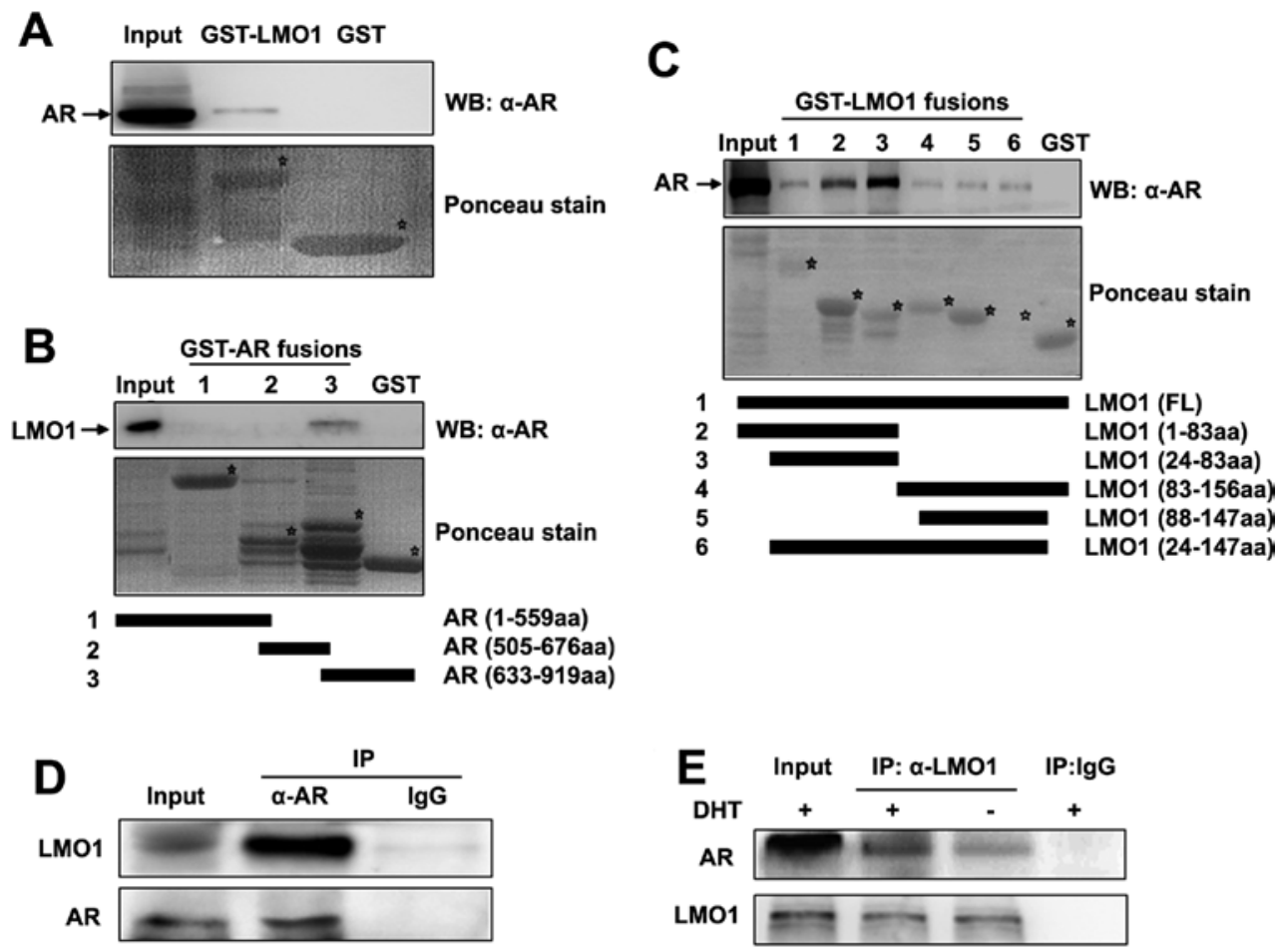

Figure 1. LMO1 interacts with AR protein. (A-C) Mapping of binding domains in LMO1 and AR for GST pull-down assay. GST, GST-LMO1 or GST-AR deletions were incubated with indicated proteins synthesized by transcription and translation kit in vitro. Bound proteins were analyzed by immunoblotting using indicated antibodies and the loading amounts of GST-fusion proteins were assessed by ponceau staining. GST served as control. An asterisk was placed to indicate the position of the specific GST-fusion proteins. (D and E) The interaction between endogenous LMO1 and AR in CWR22RV1 cells with or without DHT, analyzed by CoIP using LMO1 and AR antibodies and precipitates were immunoblotted with indicated antibodies.

China Medical University. The histological grade of cancers was based on the criteria set by the World Health Organization.

Immunohistochemisty was previously specified (14), and the intensity values of immunohistochemical results were determined by HSCORE (histological score) (15). The HSCORE was calculated using two indices of proportion (Pí) and intensity (í). The proportion (Pí) represented the percentage of positive cells. The intensity (í) was classified as 0 (no staining), or $1+$ (light brown staining), or 2+ (brown staining) or $3+$ (heavy brown staining). HSCORE was obtained for each slide by using the following algorithm: HSCORE $=\sum$ Píxí. Where $i ́$ is $0,1,2,3$ and Pí varies from 0.0 to 1.0, HSCOREs ranged from a minimum of zero in cases with no staining to a maximum of 3.0 in cases in which all the cells stained with maximal intensity. The HSCORE was determined by two independent observers.

Luciferase assay. Luciferase assay has been previously described (16). CV1, PC-3 and CWR22RV1 cells were transfected with indicated reporter and Renilla-encoding plasmids with or without DHT. Then, the supernatants from cells were measured for luciferase activities using the Dual-luciferase reporter assay system (Promega) and a Berthold Luminometer LB9570 (EG \& G. Berthold, Freiburg, Germany). Measured luciferase values were normalized to internal Renilla control. Each experiment was repeated in triplicate.

Real-time PCR. Real-time PCR has been described (17). Total RNA was isolated from CWR22RV1 cells using TRIzol reagent (Invitrogen). cDNA was synthesized by reverse transcription using an $\mathrm{RT}$ reaction kit (Takara) according to the manufacturer's instruction. Real-time PCR was performed using LightCycler 480 (Roche) and SYBR ${ }^{\circledR}$ Premix ExTaq (Takara) as a DNA-specific fluorescent dye. The housekeeping gene $\beta$-actin was used as an endogenous control. PCR was performed in triplicate for each reaction. The sequences of PCR primers were as follows: P21 forward, 5'-TGTCCGTCAG AACCCATGC-3' and $P 21$ reverse, 5'-AAAGTCGAAGTTCCA TCGCTC-3'; PSA forward, 5'-CACCTGCTCGGGTGATTC TG-3' and PSA reverse, 5'-CCACTTCCGGTAATGCACCA-3'.

Statistical analysis. All statistical calculations were performed using the SPSS (16.0) statistical software (SPSS, Inc., Chicago, IL, USA). Immunohistochemistry data were analyzed by one-way ANOVA. For luciferase assay and real-time PCR, two-sided Student's t-test was used to determine the significant difference. Data are presented as means \pm standard errors (SE). Statistical significance was accepted at $\mathrm{P}<0.05$.

\section{Results}

Identification of LMOI as an interacting protein of AR. To investigate the potential physical and functional interactions between LMO1 and AR, as a first step, we performed in vitro GST pull-down assays to demonstrate the ability of LMO1 and AR to interact directly. An interaction between AR and GST-LMO1 was observed (Fig. 1A), suggesting that LMO1 is an AR-binding protein.

To define the interacting domains within AR that were required for the observed interaction, we used three deletion 


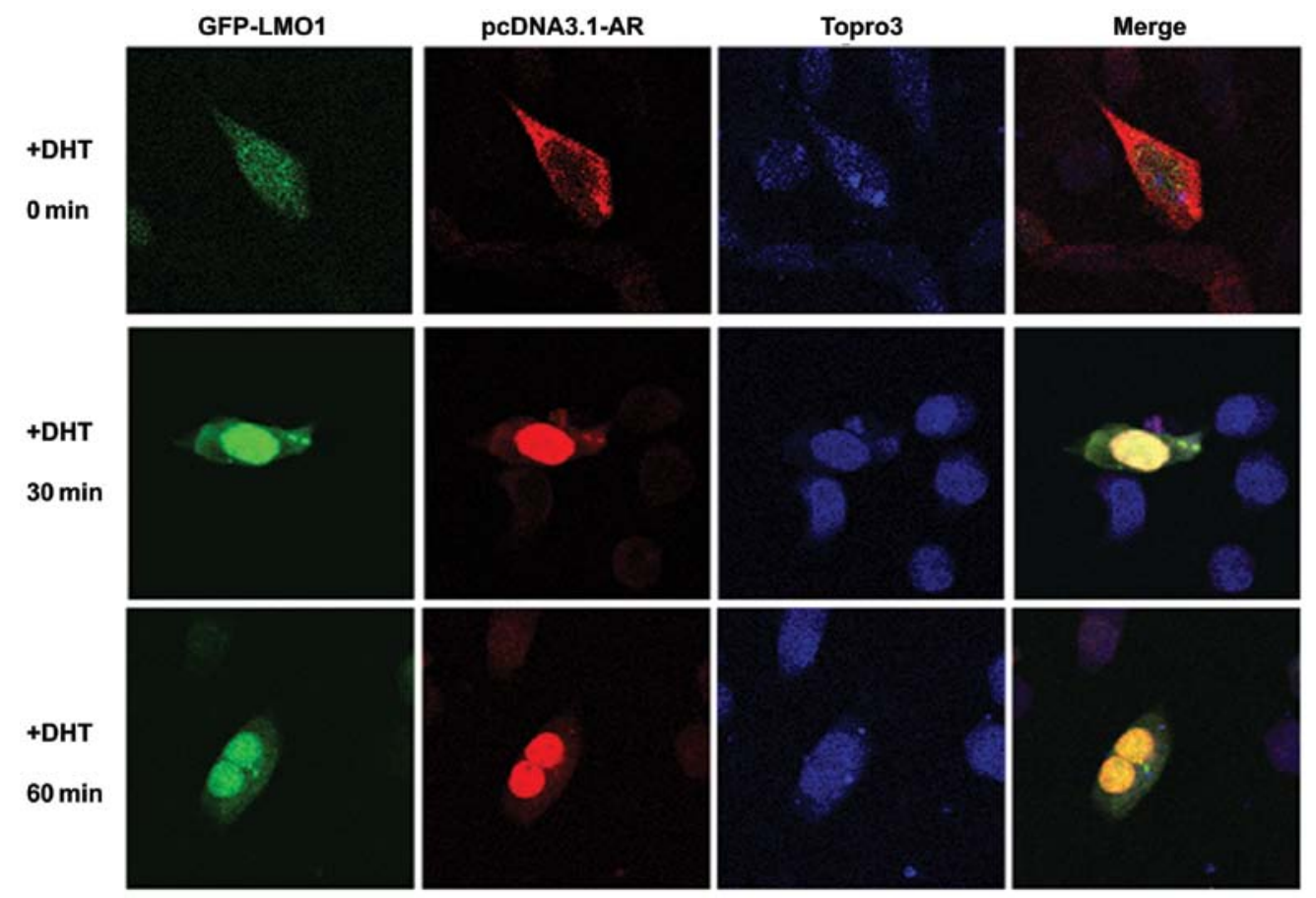

Figure 2. The localization of LMO1 and AR in cells. HEK293 cells were transfected with GFP-LMO1 and AR constructs. After starvation with steroid hormone for $16 \mathrm{~h}$, cells were stimulated with DHT for 0,30 and $60 \mathrm{~min}$ as indicated. Then they were fixed and incubated with anti-AR antibody followed by Alexa Flour 546 (red) antibody, and subsequently subjected to confocal immunofluorescence microscopy. Topro3 (blue) stains revealed the positions of the nucleus.

constructs of AR (NTD, DBD and LBD) in GST pull-down assays. In vitro-translated LMO1 was bound to LBD domain rather than NTD or DBD domain (Fig. 1B). The results indicate that AR interacts with LMO1 primarily via its LBD.

We then identified the AR-binding domain in LMO1 using a series of LMO1 deletion constructs. GST pull-down assays showed that deletion of either of the LMO1 LIM domains would not abolish the ability of the LMO1 binding to the AR (Fig. 1C), which suggests that both of the LIM domains of LMO1 are required for the cooperation with AR.

Subsequently, immunoprecipitation assays were used to show the interaction of endogenous LMO1 and AR in the AR-positive PCa cell line CWR22RV1. Cell lysates were subjected to immunoprecipitate with AR antibody and then underwent immunoblotting analysis with antibodies to LMO1 and AR. Endogenous LMO1 was immunoprecipitated by the AR antibody, but not by the control IgG antibody, confirming the physiological association between endogenous LMO1 and AR (Fig. 1D). Furthermore, the same experiments carried out in a reciprocal manner revealed that endogenous AR was also immunoprecipitated by the LMO1 antibody (Fig. 1E). In addition, the interaction was significantly enhanced in the presence of DHT. Taken together, our findings suggest that LMO1 can interact with AR both in vitro and in vivo.

LMOI cotranslocates into the nucleus with AR in an androgendependent manner. To observe the intracellular localization of LMO1 and AR, we cotransfected HEK293 cells with GFP-LMO1 and pcDNA3.1-AR, followed by treatment with DHT. LMO1 was primarily distributed in both cytoplasmic and nuclear compartments, and then became concentrated in the nucleus after DHT treatment (Fig. 2, first column) from immunofluorescence analysis. As we know, AR translocated into the nucleus upon DHT exposure (Fig. 2, second column). Interestingly, merged images revealed that the distribution of LMO1 and AR in the nucleus overlapped under DHT treatment (Fig. 2, fourth column). Together, the results suggest that LMO1, as part of an AR complex, cotranslocates into the nucleus with AR in an androgen-dependent manner, thus, probably modulating AR transcriptional activity.

Expression of LMO1 in clinical prostate biopsies. To test the role of LMO1 expression in PCa progression, we observed the expression of LMO1 in clinical prostate biopsies including 64 cases of $\mathrm{PCa}$ and 33 cases of benign prostatic hyperplasia (BPH) by immunohistochemical staining. The representative images are shown in Fig. 3A. Higher staining of LMO1 was observed in PCa tissues than in BPH tissues (Fig. 3B). Moreover, there was an increase in LMO1 staining in poorly differentiated PCa (Gleason score 8-10) compared with well and moderately differentiated PCa (Gleason score 2-7) (Fig. 3B). These data indicate a connection between LMO1 expression and the development of $\mathrm{PCa}$.

\section{LMO1 upregulates AR-mediated transcriptional activation} in a DHT-dependent manner. AR is an important transcription factor that regulates expression of a variety of target genes that harbor the androgen response element (ARE) in their promoters. To reveal the functional role of LMO1 in AR signaling, we first assessed the effect of its expression on the ARE luciferase activity via luciferase assays in CV1, CWR22RV1 and PC-3 cells, respectively. Cells were transfected with ARE-Luc, pRL-TK and LMO1. AR-deficient CV1 and PC-3 cells were co-transfected with AR. LMO1 induced ARE luciferase activity only in the presence of DHT stimulation. LMO1 upregulated ARE luciferase activity in a 
A

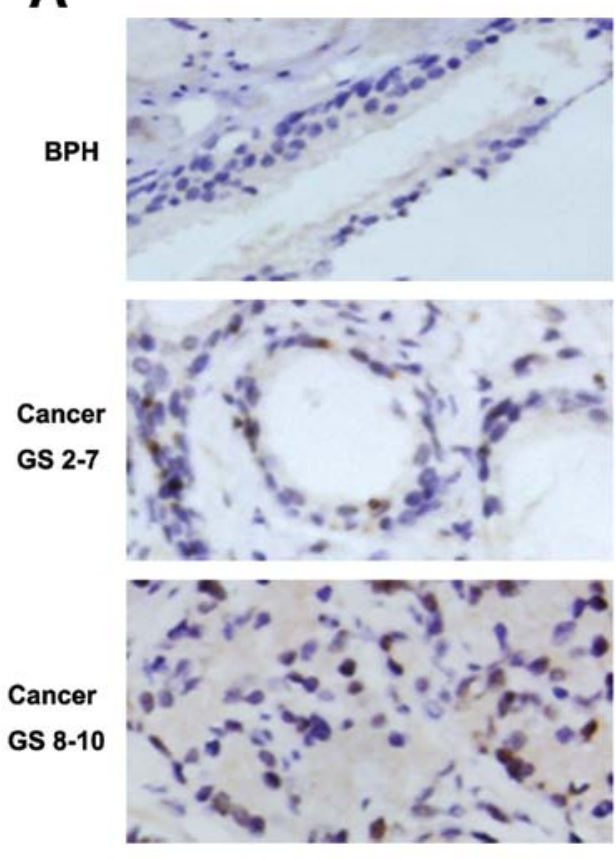

B

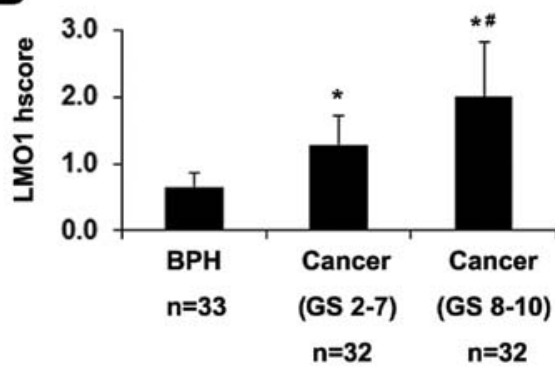

Figure 3. Immunohistochemistry of LMO1 and AR in clinical specimens. (A) Representative images of immunohistochemical staining from $\mathrm{BPH}$ prostate epithelium, lower-grade (Gleason score 2-7) and higher-grade PCa (Gleason score 8-10). (B) The quantification of the data is shown in the bar graphs. Data represents the mean values $( \pm \mathrm{SD})$ of HSCORE values, as described in Materials and methods. Data were analyzed by one-way ANOVA. ${ }^{*} \mathrm{P}<0.01$, indicates significant differences compared to the $\mathrm{BPH}$ group. ${ }^{\#} \mathrm{P}<0.01$, indicates significant differences compared to the lower-grade PCa group. GS, Gleason score.

dose-dependent manner and the increase reached a plateau at 300 ng LMO1 (Fig. 4A). LMO1 also increased ARE luciferase activity in the presence of DHT in PC-3 and CWR22RV1 cells (Fig. 4B and C). On the other hand, ARE luciferase activity was decreased when LMO1 was silenced by siLMO1 in CWR22RV1 cells (Fig. 4D).

Prostate-specific antigen (PSA) gene, another target gene downstream of AR, is known to contain an ARE, and its transcriptional level is regulated by AR (18). Furthermore, we checked PSA luciferase and found LMO1 also promoted PSA luciferase activity in CWR22RV1 and PC-3 cells, respectively (Fig. 4E and F).

LMO1 facilitates the expression of AR-targeted gene P21 and $P S A$. To further investigate the significance of LMO1 effect on AR signaling, we examined expression of the AR target genes P21 (Waf1/Cip1) and PSA, which have been implicated
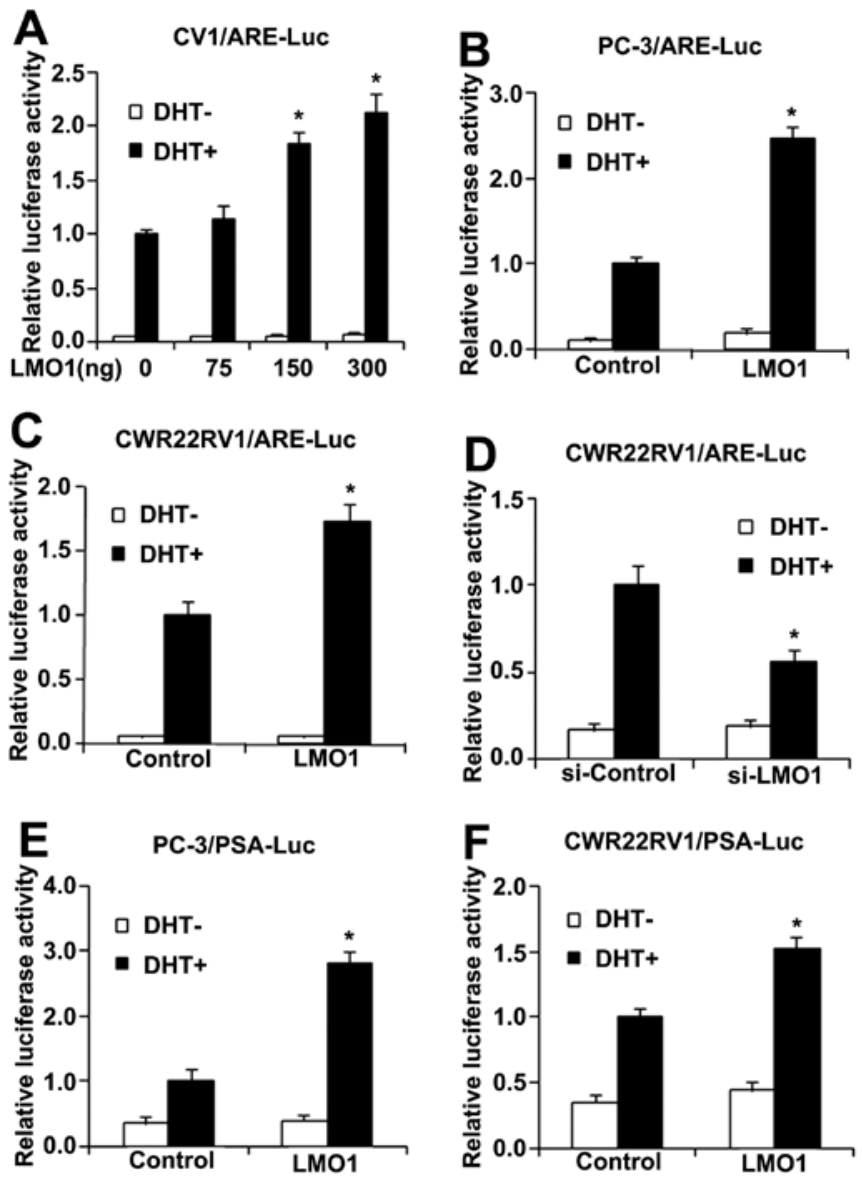

Figure 4. LMO1 stimulate AR transactivation DHT-dependently. (A-C) CV1, PC-3 or CWR22RV1 cells were transfected with ARE-Luc, pRL-TK, together with the indicated expression plasmids with or without DHT. AR-deficient CV1 and PC-3 cells were co-transfected with AR. The AREpromoter activities were measured by luciferase assays. The expression of LMO1 was evaluated by western blot assays. (D) The transcriptional activity of ARE was estimated in CWR22RV1 cells transfected with ARE-Luc, pRL-TK, together with LMO1-siRNA or control, with or without DHT. The expression of LMO1 was evaluated by western blot assays. (E and F) PC-3 or CWR22RV1 cells were transfected with PSA-Luc, pRL-TK together with vector or Myc-LMO1 constructs, with or without DHT, and then assayed for luciferase activity. Experiments were repeated three times $(n=3)$. ${ }^{*} \mathrm{P}<0.05$, indicates significant differences compared to the control group.

in prostate cancer cells $(19,20)$. When CWR22RV1 cells were transiently transfected with Myc-LMO1, the protein (Fig. 5A) and mRNA levels (Fig. 5B) of P21 and PSA increased, respectively. Furthermore, the expression of protein (Fig. 5C) and mRNA (Fig. 5D) of P21 and PSA were elevated, respectively, in CWR22RV1 cells stably transfected with Myc-LMO1 compared to cells with the vector. On the other hand, when LMO1 levels were repressed by transfection with siLMO1, the protein (Fig. 5E) and mRNA levels (Fig. 5F) of P21 and PSA decreased accordingly.

\section{Discussion}

One of the most troubling aspects of PCa progression is that most cases will ultimately progress into a hormone-refractory stage with high mortality rates after an initial response to androgen-deprivation therapy. Despite advances in the detection and treatment of $\mathrm{PCa}$, current therapies remain limited 


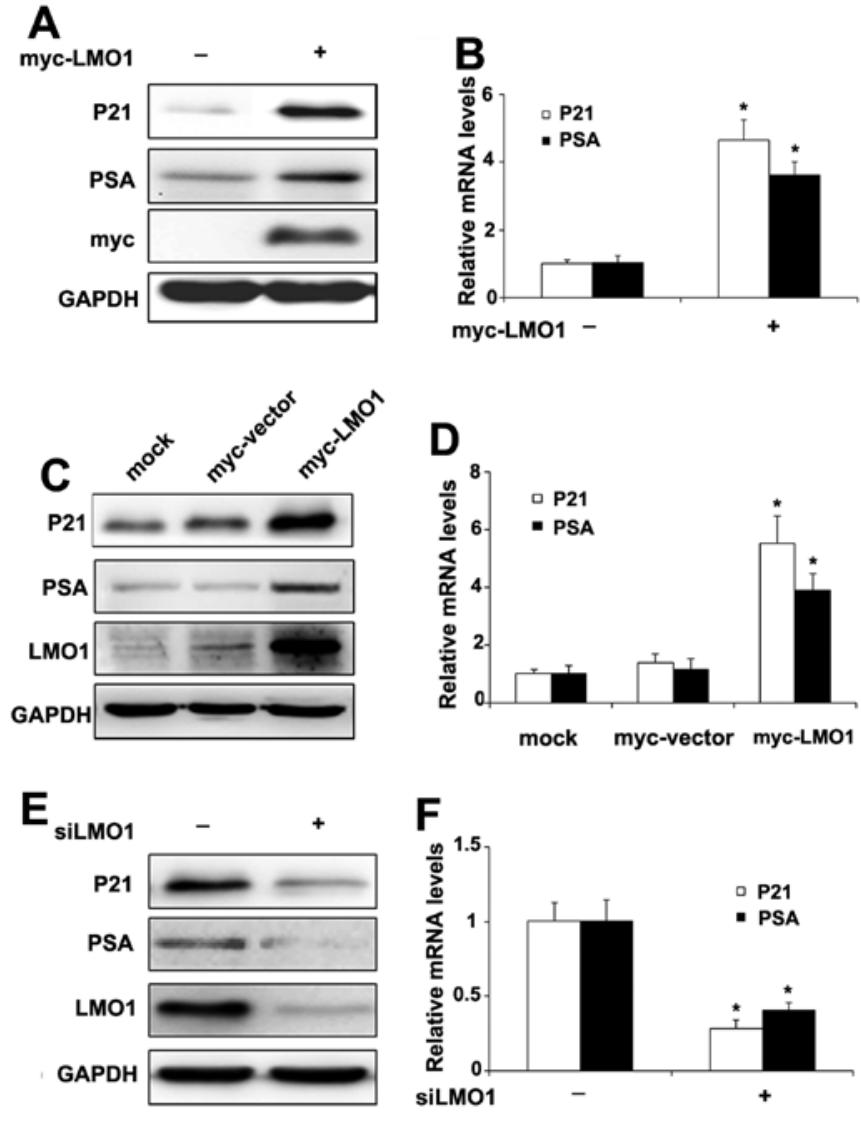

Figure 5. Effect of LMO1 on AR-targeted gene P21 and PSA in PCa cells. (A-D) Overexpression of LMO1 promotes the expressions of P21 and PSA. After CWR22RV1 cells were transiently transfected with vector or Myc-LMO1 constructs, the protein (A) or mRNA (B) levels of P21, PSA and myc were estimated by western blot analysis or real-time PCR analysis. Western blot assays or real-time PCR analysis were carried out to estimate the (C) protein and (D) mRNA levels of P21 and PSA in CWR22RV1 cells stably transfected with vector or Myc-LMO1 constructs. (E and F) Knockdown of LMO1 inhibits expression of P21 and PSA. siLMO1 or siControl were transfected into CWR22RV1 cells. Experiments were repeated three times $(n=3)$. ${ }^{*} \mathrm{P}<0.05$, indicates significant differences compared to the control group.

for the development of androgen-independent state (21). This highlights the need for a better understanding of the mechanism of constitutive activation of AR-mediated growth and subsequent androgen-independent receptor activation involved in PCa progression, aiming to develop novel targets for the diagnosis and treatment of the disease. Both the AR and its coregulators have been extensively studied as hopeful therapeutic targets for the treatment of PCa (22).

In the present study, we first provide evidence that LMO1, which is overexpressed in human PCa tissue, is an AR-interacting protein serving as a coactivator of AR to promote the AR-mediated transactivation function and subsequently amplify the expressions of P21 and PSA. These findings indicate that LMO1 is involved in PCa progression by acting as a coactivator of AR.

LMO proteins are emerging as crucial molecules in a wide variety of human cancers. All four LMO family members play key roles in several cancers. LMO1 and LMO2 are involved in the onset of T cell leukemia. LMO1 was identified near the break point of the chromosomal translocation $t(11 ; 14)$ (p15;q11) in a T-ALL cell line $(8,23)$. LMO2 was subsequently found at the junction of the chromosomal translocation $\mathrm{t}(11 ; 14)$ (p13;q11) in T-ALL $(24,25)$. Even in the absence of chromosomal lesions near LMO genes, overexpression of LMO1 or LMO2 is found in $\sim 50 \%$ of human T-ALLs (26). The roles of LMO1- and LMO2-transgenic mice during the development of T-ALL have been more clearly defined $(27,28)$. Besides, LMO1 and LMO3 are implicated in human neuroblastoma. Silence of LMO1 may suppress the growth of neuroblastoma cells with high LMO1 expression, whereas overexpression of LMO1 in neuroblastoma cells with low LMO1 expression promotes proliferation (29). The overexpressing LMO3 shows a marked increase in cell growth and is indicative of poor prognosis in neuroblastoma (30). LMO4 seems to regulate progression of breast cancer by modulating cell cycle arrest, cell proliferation, and apoptosis $(31,32)$.

In the present study, the overexpression of LMO1 was detected in human PCa tissues and it contributed to advanced stage of $\mathrm{PCa}$. Ma et al (33) reported that LMO2 expression was significantly higher in PCa than in normal epithelium and correlated positively with the level of aggressiveness in human PCa. Yet no data on LMO1 expression in human PCa are available to date. For the first time, our data demonstrated that overexpression of LMO1 was found in human PCa tissues, which also correlated with the level of cancer stage. Moreover, there have been no data revealing the cellular location of LMO1 in PCa cells. Our immunofluorescence analysis in the present study demonstrated that LMO1 was distributed in both the nucleus and the cytoplasm and then cotranslocated into the nucleus with AR after DHT treatment.

AR cofactors that associate with AR and influence its transcriptional activity could contribute to $\mathrm{PCa}$ progression. Here, we first demonstrated LMO1 interacted with AR to form a complex, then cotranslocated into the nucleus with $\mathrm{AR}$ in an androgen-dependent manner and enhanced the AR transcriptional activity. It is known that P21 and PSA are the target genes of AR in prostate and their expressions are linked to the progression of prostate cancer. P21, which belongs to a second family of cyclin-dependent-kinase inhibitors, has various biological functions such as cell cycle control, DNA repair and anti-apoptosis $(34,35)$. Androgens can upregulate the expression of P21 at both the mRNA and protein levels in cell lines $(19,36)$. Increased expression of P21 is associated with advanced PCa or cancer relapse in patients $(37,38)$. P21 protects cells against p53-induced apoptosis, rescuing cells from a path of programmed cell death to one of enhanced survival (39). PSA is highly expressed in prostate gland and is one of the most reliable diagnostic markers for PCa (40). Our results support that LMO1 is also able to induce the expression of P21 and PSA in mRNA and protein levels. These results imply that LMO1 acts as a cofactor to modulate expression of some AR-target genes, thus regulating PCa development and progression.

In summary, our results indicate that LMO1 binds to $\mathrm{AR}$ and co-localize with AR in the nucleus, serving as a coactivator of AR to promote the AR-mediated transactivation function and subsequently induce the expression of P21 and PSA, downstream targets of AR. In addition, the expression of LMO1 in PCa tissues was significantly higher than that in BPH tissues and their expression levels positively correlated with the grade of cancers. This finding provides insights into 
the role of LMO1 in the progression of PCa. Therefore, LMO1 could be a potential prognostic marker and drug target for human prostate cancer.

\section{Acknowledgements}

We thank Dr Yue Zhao (China Medical University) for providing the plasmids. We would like to express our gratitude to all the subjects who participated in the present study.

\section{References}

1. Siegel RL, Miller KD and Jemal A: Cancer statistics, 2015. CA Cancer J Clin 65: 5-29, 2015.

2. Yap TA, Zivi A, Omlin A and de Bono JS: The changing therapeutic landscape of castration-resistant prostate cancer. Nat Rev Clin Oncol 8: 597-610, 2011.

3. Zhou Y, Bolton EC and Jones JO: Androgens and androgen receptor signaling in prostate tumorigenesis. J Mol Endocrinol 54: R15-R29, 2015.

4. Gelmann EP: Molecular biology of the androgen receptor. J Clin Oncol 20: 3001-3015, 2002.

5. Heinlein CA and Chang C: Androgen receptor in prostate cancer. Endocr Rev 25: 276-308, 2004

6. Heemers HV and Tindall DJ: Androgen receptor (AR) coregulators: A diversity of functions converging on and regulating the AR transcriptional complex. Endocr Rev 28: 778-808, 2007.

7. Chmelar R, Buchanan G, Need EF, Tilley W and Greenberg NM: Androgen receptor coregulators and their involvement in the development and progression of prostate cancer. Int J Cancer 120: 719-733, 2007.

8. Boehm T, Foroni L, Kennedy $M$ and Rabbitts $\mathrm{TH}$ : The rhombotin gene belongs to a class of transcriptional regulators with a potential novel protein dimerisation motif. Oncogene 5: 1103-1105, 1990.

9. Jurata LW and Gill GN: Structure and function of LIM domains. Curr Top Microbiol Immunol 228: 75-113, 1998.

10. Dawid IB, Breen JJ and Toyama R: LIM domains: Multiple roles as adapters and functional modifiers in protein interactions Trends Genet 14: 156-162, 1998.

11. Matthews JM, Lester K, Joseph S and Curtis DJ: LIM-domainonly proteins in cancer. Nat Rev Cancer 13: 111-122, 2013.

12. Rabbitts TH: LMO T-cell translocation oncogenes typify genes activated by chromosomal translocations that alter transcription and developmental processes. Genes Dev 12: 2651-2657, 1998.

13. Liu T, Li Y, Gu H, Zhu G, Li J, Cao L and Li F: p21-Activated kinase 6 (PAK6) inhibits prostate cancer growth via phosphorylation of androgen receptor and tumorigenic E3 ligase murine double minute-2 (Mdm2). J Biol Chem 288: 3359-3369, 2013.

14. Chmelar R, Buchanan G, Need EF, Tilley W and Greenberg NM: Downregulation of p21-activated kinase-1 inhibits the growth of gastric cancer cells involving cyclin B1. Int J Cancer 125 : 2511-2519, 2009

15. Berchuck A, Soisson AP, Clarke-Pearson DL, Soper JT, Boyer CM, Kinney RB, McCarty KS Jr and Bast RC Jr: Immunohistochemical expression of CA 125 in endometrial adenocarcinoma: Correlation of antigen expression with metastatic potential. Cancer Res 49: 2091-2095, 1989.

16. Wang C, Li Y, Zhang H, Liu F, Cheng Z, Wang D, Wang G, Xu H, Zhao Y, Cao L, et al: Oncogenic PAK4 regulates Smad2/3 axis involving gastric tumorigenesis. Oncogene 33: 3473-3484, 2014.

17. Li Y, Shao Y, Tong Y, Shen T, Zhang J, Li Y, Gu H and Li F: Nucleo-cytoplasmic shuttling of PAK4 modulates $\beta$-catenin intracellular translocation and signaling. Biochim Biophys Acta 1823: 465-475, 2012.

18. Riegman PH, Vlietstra RJ, van der Korput JA, Brinkmann AO and Trapman J: The promoter of the prostate-specific antigen gene contains a functional androgen responsive element. Mol Endocrinol 5: 1921-1930, 1991.

19. Lu S, Liu M, Epner DE, Tsai SY and Tsai MJ: Androgen regulation of the cyclin-dependent kinase inhibitor p21 gene through an androgen response element in the proximal promoter. Mol Endocrinol 13: 376-384, 1999.

20. Cleutjens KB, van Eekelen CC, van der Korput HA, Brinkmann $\mathrm{AO}$ and Trapman J: Two androgen response regions cooperate in steroid hormone regulated activity of the prostatespecific antigen promoter. J Biol Chem 271: 6379-6388, 1996.
21. Taplin ME and Balk SP: Androgen receptor: A key molecule in the progression of prostate cancer to hormone independence. $\mathrm{J}$ Cell Biochem 91: 483-490, 2004.

22. Debes JD and Tindall DJ: Mechanisms of androgen-refractory prostate cancer. N Engl J Med 351: 1488-1490, 2004.

23. Boehm T, Baer R, Lavenir I, Forster A, Waters JJ, Nacheva E and Rabbitts TH: The mechanism of chromosomal translocation $\mathrm{t}(11 ; 14)$ involving the $\mathrm{T}$-cell receptor $\mathrm{C}$ delta locus on human chromosome $14 \mathrm{q} 11$ and a transcribed region of chromosome 11p15. EMBO J 7: 385-394, 1988.

24. Boehm T, Foroni L, Kaneko Y, Perutz MF and Rabbitts TH: The rhombotin family of cysteine-rich LIM-domain oncogenes: Distinct members are involved in T-cell translocations to human chromosomes 11p15 and 11p13. Proc Natl Acad Sci USA 88: 4367-4371, 1991.

25. Van Vlierberghe $\mathrm{P}$, van Grotel M, Beverloo HB, Lee C, Helgason T, Buijs-Gladdines J, Passier M, van Wering ER, Veerman AJ, Kamps WA, et al: The cryptic chromosomal deletion del(11)(p12p13) as a new activation mechanism of LMO2 in pediatric T-cell acute lymphoblastic leukemia. Blood 108: 3520-3529, 2006.

26. Ferrando AA, Neuberg DS, Staunton J, Loh ML, Huard C, Raimondi SC, Behm FG, Pui CH, Downing JR, Gilliland DG, et al: Gene expression signatures define novel oncogenic pathways in $\mathrm{T}$ cell acute lymphoblastic leukemia. Cancer Cell 1: 75-87, 2002.

27. Tremblay M, Tremblay CS, Herblot S, Aplan PD, Hébert J, Perreault $\mathrm{C}$ and Hoang T: Modeling T-cell acute lymphoblastic leukemia induced by the SCL and LMO1 oncogenes. Genes Dev 24: 1093-1105, 2010.

28. Neale GA, Rehg JE and Goorha RM: Disruption of T-cell differentiation precedes T-cell tumor formation in LMO-2 (rhombotin-2) transgenic mice. Leukemia 11 (Suppl 3): 289-290, 1997.

29. Wang K, Diskin SJ, Zhang H, Attiyeh EF, Winter C, Hou C, Schnepp RW, Diamond M, Bosse K, Mayes PA, et al: Integrative genomics identifies LMO1 as a neuroblastoma oncogene. Nature 469: 216-220, 2011.

30. Aoyama M, Ozaki T, Inuzuka H, Tomotsune D, Hirato J, Okamoto Y, Tokita H, Ohira M and Nakagawara A: LMO3 interacts with neuronal transcription factor, HEN2, and acts as an oncogene in neuroblastoma. Cancer Res 65: 4587-4597, 2005.

31. Montañez-Wiscovich ME, Seachrist DD, Landis MD, Visvader J, Andersen B and Keri RA: LMO4 is an essential mediator of ErbB2/HER2/Neu-induced breast cancer cell cycle progression. Oncogene 28: 3608-3618, 2009.

32. Wang N, Lin KK, Lu Z, Lam KS, Newton R, Xu X, Yu Z, Gill GN and Andersen B: The LIM-only factor LMO4 regulates expression of the BMP7 gene through an HDAC2-dependent mechanism, and controls cell proliferation and apoptosis of mammary epithelial cells. Oncogene 26: 6431-6441, 2007.

33. Ma S, Guan XY, Beh PS, Wong KY, Chan YP, Yuen HF, Vielkind $J$ and Chan KW: The significance of LMO2 expression in the progression of prostate cancer. J Pathol 211: 278-285, 2007.

34. Levine AJ: p53, the cellular gatekeeper for growth and division. Cell 88: 323-331, 1997.

35. Wang $\mathrm{J}$ and Walsh K: Resistance to apoptosis conferred by Cdk inhibitors during myocyte differentiation. Science 273: 359-361, 1996.

36. Lu S, Tsai SY and Tsai MJ: Molecular mechanisms of androgenindependent growth of human prostate cancer LNCaP-AI cells. Endocrinology 140: 5054-5059, 1999.

37. Omar EA, Behlouli H, Chevalier $\mathrm{S}$ and Aprikian AG: Relationship of $\mathrm{p} 21$ (WAF-I) protein expression with prognosis in advanced prostate cancer treated by androgen ablation. Prostate 49: 191-199, 2001.

38. Fizazi K, Martinez LA, Sikes CR, Johnston DA, Stephens LC, McDonnell TJ, Logothetis CJ, Trapman J, Pisters L, et al: The association of $\mathrm{p} 21^{(\mathrm{WAF}-1 / \mathrm{CIPl})}$ with progression to androgen-independent prostate cancer. Clin Cancer Res 8: 775-781, 2002.

39. Gorospe M, Cirielli C, Wang X, Seth P, Capogrossi MC and Holbrook NJ: p21 $1^{\text {Waf } 1 / C i p 1}$ protects against p53-mediated apoptosis of human melanoma cells. Oncogene 14: 929-935, 1997.

40. Gittes RF: Carcinoma of the prostate. N Engl J Med 324: 236-245, 1991. 